J. Appl. Cryst. (1975). 8, 185

\title{
Diffuse Scattering Studies of Defects in Irradiated Copper
}

\author{
By F. W. Young, JR and B. C. LARSON \\ Solid State Division, Oak Ridge National Laboratory, ${ }^{*}$ P. O. Box X, Oak Ridge, Tennessee 37830, U.S.A.
}

(Received 29 April 1974)

\begin{abstract}
The integral Huang diffuse X-ray scattering from crystals containing small dislocation loops can be analyzed to obtain two types of information: (1) the size distribution of the loops and (2) the total number of point defects contained in the loops. This technique has been applied to copper crystals irradiated under a number of conditions of neutron irradiation flux, dose and temperature, for which loops are produced, and to selected annealing studies of these loops, with all X-ray measurements made at room temperature. The results have been compared with electron microscopy, electrical resistivity, anomalous X-ray transmission and lattice parameter studies on similarly irradiated crystals. The size distributions were exponential-like and changed with irradiation conditions. The average size of the loops increased with irradiation temperature and with dose; there was $\sim$ factor of three increase between liquid-helium and room-temperature irradiations. After annealing to $265^{\circ} \mathrm{C}$ the distribution was truncated at the small sizes, with some increase in concentrations of the larger loops. The total number of point defects contained in the loops (measured at room temperature) was found to increase with dose but to decrease with irradiation temperature for a given dose. Assuming $2 \times 10^{-4} \Omega \mathrm{cm}$ per unit concentration, the number of point defects measured by electrical resistivity was about one-third that determined from the diffuse scattering. A comparison of the point-defect concentrations observed after irradiation and as a function of annealing will be made with existing resistivity, lattice parameter, anomalous X-ray transmission and electron microscopy measurements.
\end{abstract}

Operated by Union Carbide Corp. for the U. S. Atomic

Energy Commission.

J. Appl. Cryst. (1975). 8, 185

\section{Theory of the Scattering from Crystals Containing Strongly Distorting Defect Clusters}

\author{
BY H. TRINKAUS
}

Institut für Festkörperforschung der Kernforschungsanlage Jülich, Postfach 365, 517 Jülich, Germany (BRD)

(Received 29 April 1974)

Analytical methods to describe the scattering from crystals containing defect clusters with strong displacement fields are reviewed [e.g. Krivoglaz, M. A. (1969). The Theory of X-ray and Thermal-Neutron Scattering by Real Crystals. New York: Plenum; Trinkaus, H. (1971). Z. angew. Phys. 31, 229-235; Dederichs, P. H. (1971). Phys. Rev. B4, 1041-1050; Trinkaus, H. (1973). Z. Naturforsch. 28a, 980-993; Dederichs, P. H. (1973). J. Phys. F: Metal Phys. 3, 471]. The following aspects for classifying lattice defects are taken as the basis of the discussion: the degree of continuity (boundaries, short-range variations, distortion fields), the dimension (zero, one, two), the symmetry (point symmetry of the lattice and subgroups of it), as well as the shape (spherical, cylindrical, planar) and the structure (loose, coherent, amorphous clusters) of the defect regions. These aspects are related to typical features of the lattice correlation function and the scattering function. The correlation function for a distorted lattice is discussed in detail. Here the effect of different types of clusters on the dependency of the Debye-Waller index upon the distance vector is illustrated. The long- and short-range regions of this function are related to the characteristic regions of the scattering pattern, that is to the 'Huang scattering' and 'the asymptotic distortion scattering'. Three typical approximations are reviewed: the single-defect, the one-phonon and the Stokes-Wilson approximations. The Stokes-Wilson approximation based on local reflexions within the lattice is shown to be the most useful method to describe the scattering from strongly distorting defect clusters. It yields the steeply decreasing 'asymptotic distortion scattering' from the environs of cluster regions and, for some types of clusters, shoulders on the scattering pattern due to the cluster cores and their immediate proximities. A classification of defect clusters can be set up by referring to the positions of the centres of diffuse scattering. As an interesting example dislocation loops are considered in some more detail. What information about the type, the size and the distortion strength of defect clusters as well as about the distribution of these properties can be deduced from scattering data is discussed finally. 